\title{
TRAUMA-INFORMED CARE PRINCIPLES AND PRACTICES AT A RESIDENTIAL FACILITY FOR ADOLESCENT GIRLS
}

\author{
Natalie Zanoni \\ Lad Lake St. Rose Youth and Family Center, Milwaukee, WI
}

Creating a trauma-informed, safe environment for residents who are survivors of traumatic events and abuse is the focus of care and treatment at Lad Lake St. Rose Youth and Family Center. Trauma-informed care is embedded in the organizational philosophy, the training of all care staff, the intake processes, and treatment principles.

\section{Background}

St. Rose was founded in 1848, making it the oldest social service agency in Wisconsin. St. Rose joined Lad Lake, Inc., in 2010 and is comprised of a residential program, an onsite school, male and female group homes, and a stabilization unit. Lad Lake St. Rose Sienna residential unit serves adolescent females, ages 12-17, experiencing physical, behavioral, emotional, and psychological problems such as self-abuse, trauma-abuse, neglect, delinquent activity, running away, school failure, inappropriate sexual activity, anger control issues, and other mental health issues including depression.

Of the 30 females who were served in the residential unit during the time period of January 2013-June 2014 (18 months), 50\% were African American, 30\% Caucasian, $13 \%$ multiracial, 3\% Latina, and 3\% Native American. The average age upon admission was 15.1 years, with ages ranging from 13 to 17. Most of the residents had been in prior and often multiple placements before coming to Lad Lake St. Rose. The 30 residents had been in an average of 7.8 placements prior to admission. These include group homes (47\%), hospitalizations (37\%), secure detention $(43 \%)$, residential facilities $(27 \%)$, secure juvenile correctional facilities $(20 \%)$, shelters $(17 \%)$, foster homes $(13 \%)$, stabilization units $(3 \%)$, with other family members or relatives (3\%), and rehabilitation facilities (3\%). The girls served by Lad Lake St. Rose were more likely to have had traumatic experiences, including multiple forms of abuse and neglect as well as family dysfunction. Twenty of the 30 residents (67\%) reported sexual abuse, 25 (83\%) reported physical abuse, and 18 (60\%) reported both physical and sexual abuse.

\section{Guiding Philosophy}

A key component of the trauma-informed care philosophy of St. Rose is the use of nonviolence in all interactions with residents. Residents are encouraged to make decisions of their own volition and not because they are afraid of being punished or because they are being physically forced to do so. According to the National Alliance on Mental Illness (2003), restraint and seclusion have no therapeutic value and 
should only be used as emergency safety measures because restraint and seclusion can be detrimental to those who have experienced trauma. Many residents have been restrained during assaults and at prior placements. Restraining a resident or secluding her in a room with no stimulation or assistance can retraumatize her and make her more prone to flashbacks. Physical intervention is used only as a last resort to protect the resident or others. St. Rose is one of the only licensed residential facilities in Wisconsin that does not have a seclusion room. In contrast, according to a recent study, $76 \%$ of residential treatment facilities for children had secluded and restrained clients in the previous year (Ramirez de Arellano, Lyman, Jobe-Shields, George, \& Dougherty, 2014). Alternatively, Lad Lake St. Rose provides residents with low stimulus rooms, walks with staff, a planned serenity garden onsite, and the availability of art activities. Residents are able to participate in religious activities on a weekly basis, such as attending church services, spiritual groups, and Native American smudging rituals. Debriefing sessions with the therapist are also conducted.

\section{Training}

All direct care staff, including youth care workers, the nurse, and the teacher, receive trauma-informed care training to provide insight into those who have experienced trauma and how it influences their thoughts, feelings, and behavior. Training includes nonviolent crisis intervention provided by the Crisis Prevention Institute and Risking Connections, which presents a relational framework, RICH (Respect, Information-sharing, Connection, and Hope), and skills to use when working with survivors of traumatic experiences.

The residential therapist has a certificate in trauma counseling. General staff training on topics such as family relationships and sexual exploitation is traumainformed as well. Training provides staff with an understanding of the stigma of abuse and its effects on the resident, the reasons a resident may react to certain situations in a particular manner, and the help needed to prevent her from going into crisis.

\section{Intake}

Screening for past traumatic experiences is completed using the Adverse Childhood Experiences (ACE) tool and an initial suicide assessment. The ACE tool reveals the client's current thoughts, reactions, and dysfunctions (sleep, appetite, and social relations), and the presence of posttraumatic stress symptoms. The higher the score (on a scale of 1 to 10), the greater the exposure and the greater the risk of negative consequences. The initial suicide assessment addresses past and current self-harm behaviors, thoughts of suicide, past suicide attempts including the method used, AODA concerns, risky behaviors such as delinquency and running away, and risky sexual behaviors. The suicide assessment explores the career aspirations of the client, which speaks to a sense of future. This conveys whether the client feels hopeful 
or hopeless and indicates whether the client believes that she has the capabilities and resources to live a productive life as an adult.

The assessment by the therapist also includes exploring the client's family history with her, including her parents' or guardians' backgrounds, legal issues, mental health concerns, and AODA issues. This provides key insights into the client's past experiences and any sources of trauma. It is important to know if the client's parents are still in her life and if her parents or guardians are a reliable and stable presence. The care plan is developed with the understanding that the youth care workers will partner with the family in the treatment of the client.

The client's individualized plan identifies triggers and soothers. Knowing situations, people, and even times of the day that may trigger a strong emotional reaction in the client and subsequently that may help her to calm down provide important insights into the client's thoughts, feelings, and behaviors. Triggers and soothers are discovered by self-report and through observation by youth care workers. All youth care workers and other direct care staff must be aware of the client's triggers and soothers, especially since the client will often be triggered on the unit or in school.

\section{Treatment}

Residents participate in individual therapy sessions twice weekly and group therapy four times weekly. Equine, art, and health therapy groups are provided weekly, and most residents participate in family therapy. Residents also meet with a child or adolescent psychiatrist to monitor psychiatric medications during their placements. Residents receive round-the-clock care from youth care workers and have opportunities for recreational activities with program peers, both on the unit and in the community.

Therapists use a strength-based, empowerment approach to treatment with the intention of encouraging clients to become survivors, not victims. Therapists emphasize their role in providing assistance and support to clients and guiding them in making the necessary progress to move on from residential treatment and to ultimately succeed in life.

Symptoms of past trauma and neglect can result in clients' being unable to trust others and feel threatened by them. Special care is taken to ensure that the therapist's office is a safe and stress-free environment. There are no interruptions by phone or visitor. Due to the physical layout of the office, the door can be left open, but the session is not visible nor audible to anyone in the hallway. This is beneficial because some clients experience panic or anxiety in an enclosed room with another person. Toys and trinkets are available to provide tactile stimulation and reduce anxiety.

Therapists employ trauma-focused cognitive behavioral therapy (TF-CBT) techniques with children and adolescents who have had traumatic experiences, and research has validated the effectiveness of TF-CBT for this group. For example, 
a study of children who had experienced sexual abuse and were treated with TFCBT showed overall improvement upon completion of treatment and at 6 and 12 months after treatment (Mannarino, Cohen, Deblinger, Runyon, \& Steer, 2012). In another study, traumatized children were treated with either TF-CBT or TAU. Lower levels of posttraumatic stress symptoms, depression, and general mental health problems were reported in the children who received TF-CBT (Jensen, et al., 2014). As defined by the Substance Abuse and Mental Health Services Administration (SAMHSA), TF-CBT includes skills in psychoeducation, and parenting, relaxation, affect expression and regulation, and cognitive coping. It also includes developing a trauma narrative using in vivo exposure, attending parent and caregiver-child sessions, and enhancing safety and future development.

A TF-CBT treatment scenario involving a client who experienced sexual abuse would begin with psychoeducation, which provides specific information about the traumatic event experienced by the client. It would include information about the types of sexual abuse, why it may occur, who may be a perpetrator, how the abused person may feel and behave such as in a sexualized manner, and why the abused person would be reluctant to disclose the abuse. The therapist demonstrates relaxation techniques starting with basic but very effective controlled breathing and has the client practice them. The technique of thought-stopping is also introduced. The client is instructed to close her eyes and think of an intrusive experience. This does not need to be the most vivid or upsetting memory of the sexual abuse. After ten seconds, the client is taught to stop the thought by saying "stop" or snapping a rubber band that is around her wrist. Then the client works on replacing the intrusive thought with one that is pleasant.

The teaching of affect expression and regulation is another component of treatment. In this case the client identifies as many feelings as possible. Then the therapist helps the client to rate and differentiate between different degrees of intensity of a particular emotion. The next step is to teach the client how to express feelings appropriately in various situations. Role-playing is a good strategy in which both the client and the therapist demonstrate ways in which they have expressed feelings in real-life situations. Each role-play is reviewed, and if necessary, the therapist and client discuss different strategies for expressing feelings more appropriately.

The therapist guides the resident in creating a narrative of the sexual abuse. The narrative can be in the form of a book, picture, song, or poem. It may be necessary to do this in stages if the client is having difficulty coping with the process. The narrative is read, recited, or sung several times during subsequent therapy sessions. The resident should begin to experience progressively less extreme emotional and physiological reactivity. During the trauma narrative exercise, cognitive processing techniques are used to explore and correct cognitive distortions and errors. The therapist challenges unhelpful or inaccurate cognitions by using progressive logical questioning, also known as Socratic questioning. The questions draw 
out the inaccurate cognitions to their logical conclusions, which in most cases are undesirable or even silly.

In terms of safety and future development, clients are able to practice remaining safe in the community by going on unit outings. They also have the opportunity to interact with a diverse set of preapproved individuals who are part of Lad Lake St. Rose so that they can practice social skills and appropriate interactions.

Family therapy is an essential component of treatment. During the initial meeting, the therapist explains her role and intentions to the parents or guardians, which is to offer assistance to the family in addressing past and current issues. During therapy, the therapist serves as a mediator, especially since communication is often at the center of issues between the client and her parents or guardians. Parents and guardians are provided with psychoeduation as well, which is to assist them in helping their children. For example, the therapist teaches the parents and guardians how to use praise. Parents and guardians usually believe that they praise their children frequently, but they are often more likely to criticize their children for undesirable behavior than to praise them. Therefore, the therapist teaches parents and guardians to focus on actively praising the child for desirable behavior and ignoring undesirable behavior such as angry or defiant statements or body language, when it occurs, unless it is potentially dangerous. The therapist prepares the parents and guardians for family therapy by instructing them to use praise and ask open-ended, nonthreatening questions. They are also prepared to respond to the client's trauma narrative and are encouraged to rehearse responses to questions their child may have.

Even with preparation, family therapy can be tension-filled. Past issues may come to light that can make the parents or guardians embarrassed and resistant to addressing and processing. Parents and guardians may have personal issues such as financial problems, addiction or AODA issues, or their own mental health concerns which have impacted their relationship and any past treatment with their child. Parents and guardians tend to be protective and may be in denial regarding the actions of their child or worry that their child's actions reflect poorly on their parenting. Parents and guardians may not trust the therapist and be concerned that the therapist may provide information to the court system which may have a detrimental effect. Since it is the therapist's role to recommend how long the resident should remain in residential care, she can dictate how long the child must be separated from her parents or guardians. This can be another source of anxiety for them.

Communication between the parents or guardians and youth care workers is vital. The resident's confidentiality must be maintained while providing adequate information to the family on the progress the resident is making and any concerns that have developed while in residential care. This becomes important when the resident begins home passes. Having a strong and open communication established with the family before the resident goes home on pass will increase the likelihood that the parents and guardians will feel comfortable sharing what they 
observe during the home visits, asking for assistance with issues that may be occurring on pass, and noting any changes they may be seeing in their child.

Team meetings comprising the therapist, nurse, manager, clinical coordinator, educators, and youth care workers are held weekly. This allows the team to discuss the resident's progress and any concerns. This is essential in getting a complete picture of the resident and brings to light issues that could not be detected by the therapist alone. Therefore, all members of the care team must be involved and cognizant of issues that have resulted from past trauma. The therapist must have a positive relationship with youth care workers, which is essential to open communication and effective treatment. The resident's issues and concerns are addressed and processed in therapy for a short period of time each week.

Much of the resident's necessary change and development occurs on the residential unit and in school. Much of the real work happens on the unit and in school. The therapist also is present on the residential unit and observes the residents in school to gain an understanding of their overall functioning.

Youth care workers are in contact with the residents and provide 24 hour care to them. Trauma-informed care principles and practices are employed during each interaction that youth care workers have with residents. An awareness and understanding of the personal history and background of the resident, including any instances of trauma, are vital especially since these past experiences can affect both mood and behavior. When the youth care workers know the possible reasons and motivations for moods and behaviors, they are able to work to address them from a therapeutic standpoint rather than a punitive one. This is much more beneficial to the residents and much more effective in the successful treatment of them. Youth care workers are also sensitive to particular events, activities, or circumstances that may be impacted by past trauma. For example, bedtime seems to be a particularly challenging time. Many residents will become defiant and combative because this is typically the time when they were abused. Additionally, residents will often awaken in a terrible mood due to nightmares from the night before. Past trauma has a profound effect on the resident and all aspects of her life, and therefore, youth care workers' trauma-informed approach is essential.

Translating the principles of trauma-informed care into practice is a necessary component of the successful treatment of the clients at Lad Lake St. Rose Youth and Family Center so that they are able to heal, gain self-esteem, and develop the skills necessary to become productive members of the community. 


\section{References}

Jensen, T. K., Holt, T., Ormhaug, S. M., Egeland, K., Granly, L., Hoass, L. C., \& Wentzel-Larsen, T. (2014). Randomized effectiveness study comparing trauma-focused cognitive behavioral therapy with therapy as usual for youth. Journal of Clinical Child and Adolescent Psychology, 43(3), 356-369.

Mannarino, A. P., Cohen, J. A., Deblinger, E., Runyon, M. K., \& Steer, R. A. (2012). Trauma-focused cognitive-behavioral therapy for children: Sustained impact of treatment 6 and 12 months later. Child Maltreatment, 17(3), 231-241.

National Alliance on Mental Illness. (2003). Seclusions and restraints: Task force report. Arlington, VA: Author.

National registry of evidence-based programs and practices. Trauma-informed cognitive behavioral therapy. Retrieved from http://nrepp.samhsa.gov/ ViewIntervention. aspx?id=135

Ramirez de Arellano, M. A., Lyman, D. R., Jobe-Shields, L., George, P., \& Doughherty, R. H. (2014). Trauma-focused cognitive-behavioral therapy for children and adolescents: Assessing the evidence. Psychiatric Services, 65(5), 591-602. 